\title{
Overexpression of TEAD4 in atypical teratoid/rhabdoid tumor: New insight to the pathophysiology of an aggressive brain tumor
}

\author{
Mario Suzuki ${ }^{1,2,3}$ | Akihide Kondo ${ }^{1}$ | Ikuko Ogino $^{1}$ | Hajime Arai ${ }^{1}$ | \\ Tadanori Tomita $^{2,4}$ | Simone Treiger Sredni ${ }^{2,3,4}$
}

\begin{abstract}
${ }^{1}$ School of Medicine, Department of Neurosurgery, Juntendo University, Tokyo, Japan

${ }^{2}$ Division of Pediatric Neurosurgery, Ann and Robert H. Lurie Children's Hospital of Chicago, Chicago, Illinois

${ }^{3}$ Stanley Manne Children's Research Institute, Chicago, Illinois

${ }^{4}$ Feinberg School of Medicine, Northwestern University, Chicago, Illinois

Correspondence

Mario Suzuki, School of Medicine, Department of Neurosurgery, Juntendo University, 2-1-1 Hongo, Bunkyo-ku, Tokyo 113-8421, Japan. Email:marisuzu@juntendo.ac.jp

Grant sponsor: Juntendo University; Grant sponsor: Rally Foundation for Childhood Cancer Research; Grant number: 925540.
\end{abstract}

\begin{abstract}
Background: Atypical teratoid/rhabdoid tumor (AT/RT) is a highly malignant embryonal brain tumor that occurs mainly in early childhood. Although most of the tumors are characterized by inactivating mutations of the tumor suppressor gene, SMARCB1, the biological basis of its tumorigenesis and aggressiveness is still unknown.
\end{abstract}

Procedure: We performed high-throughput copy number variation analysis of primary cell lines generated from primary and relapsed tumors from one of our patients to identify new genes involved in AT/RT biology. The expression of the identified gene was validated in 29 AT/RT samples by gene expression profiling, quantitative real-time polymerase chain reaction, and immunohistochemistry (IHC). Furthermore, we investigated the function of this gene by mutating it in rhabdoid tumor cells.

Results: TEAD4 amplification was detected in the primary cell lines and its overexpression was confirmed at mRNA and protein levels in an independent cohort of AT/RT samples. TEAD4's coactivator, YAP1, and the downstream targets, MYC and CCND1, were also found to be upregulated in AT/RT when compared to medulloblastoma. IHC showed TEAD4 and YAP1 overexpression in all samples. Cell proliferation and migration were significantly reduced in TEAD4-mutated cells.

Conclusions: We report the overexpression of TEAD4 in AT/RT, which is a key component of Hippo pathway. Recent reports revealed that dysregulation of the Hippo pathway is implicated in tumorigenesis and poor prognosis of several human cancers. Our results suggest that TEAD4 plays a role in the pathophysiology of AT/RT, which represents a new insight into the biology of this aggressive tumor.

KEYWORDS

AT/RT, atypical teratoid/rhabdoid tumor, Hippo pathway, TEAD4, YAP1

\section{1 | INTRODUCTION}

Malignant rhabdoid tumor (MRT) is a highly aggressive pediatric embryonal tumor that can arise in any anatomic location. The most frequent sites of origin are the kidneys and brain. ${ }^{1}$ MRT that originates in the central nervous system (CNS) is called atypical teratoid/rhabdoid tumor (AT/RT). AT/RT comprises approximately 1-2\% of

Abbreviations: AT/RT, atypical teratoid/rhabdoid tumor; CNV, copy number variation; CRISPR, clustered regularly interspaced short palindromic repeats; GE, gene expression; IHC, immunohistochemistry; MB, medulloblastoma; MRT, malignant rhabdoid tumor; Q-PCR, quantitative real-time polymerase chain reaction; TEAD4, TEA domain family 4 all pediatric brain tumors, but it is the most frequent malignant brain tumor among infants. ${ }^{2,3}$ It shows a highly aggressive and unresponsive nature with a median overall survival of 6-18 months despite intensive multimodal therapy, including surgery, high-dose chemotherapy with or without intrathecal chemotherapy, and radiation therapy. ${ }^{4-6}$ Recently, reports have shown that radiation therapy and intensive multimodal chemotherapy improve the survival of patients, especially those older than 3 years of age. However, the prognosis for the majority of patients' population, especially in infants, remains still poor. ${ }^{6-10}$

Histopathologically, AT/RT is characterized by variable amounts of cells with classic rhabdoid phenotype, which shows eccentrically 
placed nuclei containing vesicular chromatin and abundant cytoplasm with eosinophilic globular inclusions. Usually, these cells with rhabdoid phenotypes are observed within areas of small undifferentiated tumor cells. Therefore, depending on the area examined, it can be misdiagnosed as other embryonal brain tumors such as medulloblastoma (MB) or a group of tumors recognized as the primitive neuroectodermal tumor of the CNS in former WHO classification. ${ }^{11,12}$ After the notable discovery of genomic alterations for AT/RT in the SMARCB1 (BAF47/hSNF5/INI1) tumor suppressor gene, which is a component of the chromatin remodeling complex switch/sucrose nonfermentable (SWI/SNF), ${ }^{13}$ negative nuclear stain for SMARCB1 protein has become the widespread procedure for diagnosis of this tumor. ${ }^{14}$ While SMARCB1 mutations are the defining genetic alterations of $A T / R T$, recent collaborative studies involving large cohorts of samples and advances in genome-wide technologies have suggested the existence of different molecular subgroups. ${ }^{15-17}$ Several groups have also explored new potential therapeutic targets. ${ }^{18-22}$ Nevertheless, much of the biology contributing to the development and aggressiveness of this tumor is still poorly understood.

TEA domain family member 4 (TEAD4) is a transcriptional factor which is a part of the Hippo signaling pathway. The Hippo pathway is conserved as a tumor suppressor pathway and plays a role in several biological processes including organ size control, tissue regeneration, cancer development, stem cell self-renewal, and differentiation. ${ }^{23,24}$ The pathway consists of two serine/threonine kinases, MST and LAT; the transcriptional co-activators, YAP1 and TAZ; and the transcription factors, TEAD1-TEAD4. When the Hippo pathway is activated, the activity of YAP1 is inhibited and the expression of its downstream genes is suppressed. Conversely, when the pathway is inactivated, YAP1 accumulates in the nucleus and forms complexes with TEADs and other transcription factors, promoting cell proliferation and cell survival and inhibiting apoptosis. ${ }^{25}$ Recently, several studies have found that mutations and altered expression of a subset of the Hippo signaling pathway genes are involved in increased cell proliferation in diverse types of human cancers such as melanoma, ovarian, breast, gastric, and colorectal cancers. Some of these reports suggest that the dysregulation of this pathway correlates with poorer prognosis. ${ }^{24,26-29}$

To clarify the aggressiveness of this tumor, we performed genomewide studies in samples from our patients including primary and relapsed tumors after interventions and found the amplification of TEAD4. Then, we validated the overexpression at both mRNA and protein levels in a larger set of samples. Its transcriptional co-activator YAP1 and downstream targets were also found to be upregulated in AT/RT, which may indicate the active status of TEAD4. Furthermore, we detected decrease in cell proliferation and migration in a TEAD4mutated rhabdoid tumor cell line. Those facts suggest that this pathway may have a key role on this tumor's biology.

\section{I MATERIALS AND METHODS}

\section{1 | Tumor samples}

Tumor samples including fresh frozen tumor tissues and formalin-fixed and paraffin-embedded (FFPE) tissue sections were collected from patients diagnosed with AT/RT and MB. The diagnoses were confirmed pathologically according to the current WHO criteria. ${ }^{11,12}$ Tumors were provided by the Juntendo University Hospital (Tokyo, Japan), Falk Brain Tumor Bank (Chicago, IL, USA), and Center for Childhood Cancer, Biopathology Center (Columbus, OH, USA), which is a section of Cooperative Human Tissue Network of The National Cancer Institute (Bethesda, MD, USA). Written informed parental consents were obtained prior to sample collection. This study was approved by the institutional review boards of Juntendo University (IRB \#2010014) and Ann and Robert H. Lurie Children's Hospital of Chicago (IRB \#2009-13778). Primary AT/RT samples from 29 patients, 4 samples from relapsed tumor tissues (Table 1), and $15 \mathrm{MBs}$ were included in our studies.

\section{2 | Primary cell culture}

Primary AT/RT cell lines were established from the primary and relapsed tumors from one of our patients. Tumor tissues were obtained at surgery, and minced in a Petri dish, and then maintained in Neurobasal-A Medium with 2\% B-27 supplement serum free, EGF, FGF-Basic, and Penicillin-Streptomycin-Glutamine (Thermo Fisher Scientific, USA) at $37^{\circ} \mathrm{C}, 5 \% \mathrm{CO}_{2} \cdot{ }^{30}$ Cellblocks were made using Array Jelly (Youken-Science Co., Ltd., Japan) according to the manufacture's protocol.

\subsection{Copy number variations (CNVs)}

Genomic DNA was isolated from both primary cell lines as described above and from the correspondent relapsed tissue using Gentra Purgene Tissue Kit (Qiagen, Germany) according to the manufacture's protocol.

A total of $250 \mathrm{ng}$ of genomic DNA was used to investigate genomic alterations using the Genome-Wide Human CytoScan HD Array (Affymetrix, USA) according to the manufacture's protocol. The data were analyzed with Affymetrix $(\mathbb{R}$ Chromosome Analysis Suite v1.2 (Affymetrix).

\section{4 | In situ hybridization}

In situ hybridization was performed on FFPE sections of the primary and relapse samples from which the primary cell lines were established, using GeneticLab QuantiGene ViewRNA kit (Affymetrix). After deparaffinization, sections were boiled in pretreatment solution for $20 \mathrm{~min}$, digested with protease for $20 \mathrm{~min}$, and then hybridized with designed probes against TEAD4 (VX1-99999-01) and YAP1 (VX699999-01). Fast Blue and Fast Red substrates were used to produce signals.

\subsection{Gene expression (GE) profiling}

Total RNA was isolated using Trizol Reagent (Invitrogen, USA) from frozen tumor tissues. GE profiling was performed using Illumina HT12 BeadChip whole-genome expression arrays (Illumina, USA). All RNA samples were treated with DNase. In vitro transcription was completed in order to synthesize biotin-labeled cDNA. A total of $1.5 \mu \mathrm{g}$ of cDNA was hybridized to each array using standard Illumina protocols. 
TABLE 1 Summary of AT/RT patients and experiments

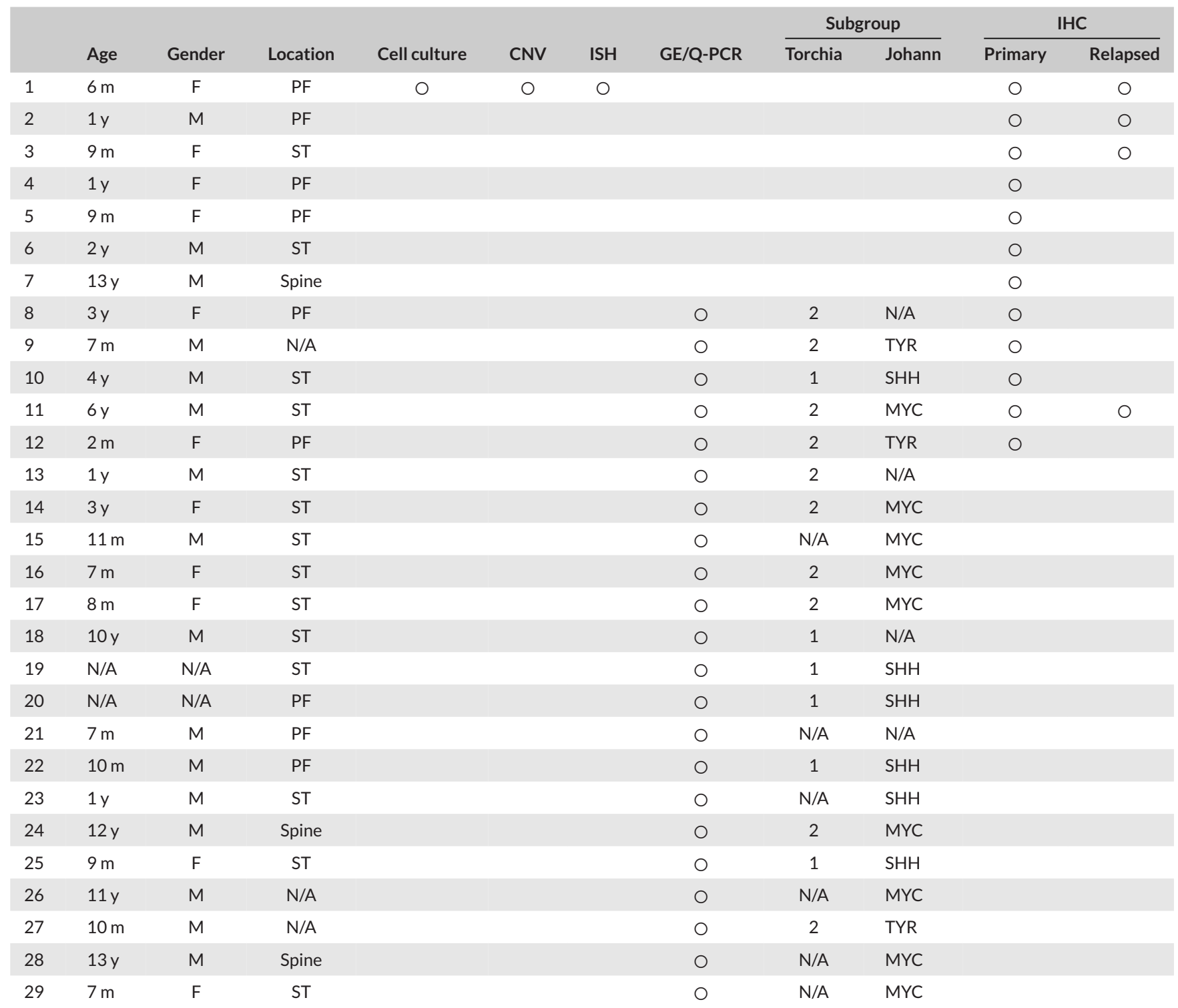

CNV, copy number variation; GE, gene expression; IHC, immunohistochemistry; ISH, in situ hybridization; m, months; N/A, not available; PF, posterior fossa; $\mathrm{Q}-\mathrm{PCR}$, quantitative real-time polymerase chain reaction; ST, supra-tentorial; $y$, years.

Slides were scanned and analyzed using BeadStudio (Illumina). Data were normalized using the quantile normalization procedure from the bioconductor package, affy (www. bioconductor. org). Kyoto Encyclopedia of Genes and Genomes-KEGG (http://www.kegg.jp/kegg/) was referred to identify enriched biological functions.

\subsection{Quantitative real-time PCR (Q-PCR)}

A total of 1,000 ng of RNA was used to make cDNA using the highcapacity RNA-to-cDNA Kit (Life Technologies, USA). The expression of selected genes was validated by TaqMan GE assays (Life Technologies). The following genes were tested: TEAD4 (Hs01125032_m1), YAP1 (Hs00902712_g1), MYC (Hs00153408_m1), and CCND1 (Hs0076553_m1). The normalized expression levels were calculated by the $\Delta \Delta \mathrm{Ct}$ method using the housekeeping gene GAPDH (Hs02758991_g1) as a reference.
Q-PCR for CNVs was performed using TaqMan Copy Number Assays (Life Technologies) according to the manufacture's protocol. Three TEAD4 probes were tested (Hs01275079_cn, Hs00784753_cn, and $\mathrm{Hs} 01667625$ _n) and RNase $\mathrm{P}$ was used as a reference. The data were analyzed with Copy Caller Software (Applied Biosystem, USA).

\section{7 | Immunohistochemistry (IHC)}

FFPE tumor tissue sections were stained using standard immunohistochemical methods with the following antibodies: polyclonal hSNF5 antibody (1:200; Novus Biologicals, USA), polyclonal TEAD4 antibody (1:200; Abcam, UK), monoclonal YAP1 antibody (1:200; Abnova, Taiwan), polyclonal Ki-67 antibody (1:200, Thermo Scientific), and polyclonal Phospho-Histone H3 antibody (PHH3) (1:5,000, Abcam). Slide interpretation was performed independently by two investigators in a blinded fashion (MS and STS). 


\section{8 | Western blotting}

After cells were lysate, protein concentration was calculated using Pierce BCA Protein Assay Kit (Thermo Fisher Scientific). A total of $20 \mu \mathrm{g}$ of proteins were loaded onto an sodium dodecyl sulfate gel and transferred to a polyvinylidene difluoride membrane. The following antibodies were used for protein detection: monoclonal TEAD4 antibody (1:1,000, Abcam) and monoclonal GAPDH loading control antibody (1:25,000, Thermo Fisher Scientific). Protein levels were detected by ECL Detection Solution (Thermo Fisher Scientific) and visualized on Bio-Rad ChemiDoc MP (Bio-Rad, USA).

\section{9 | In vitro genome edition}

We used Lentiviral-CRISPR/Cas9 (where CRISPR is clustered regularly interspaced short palindromic repeats) system to mutate TEAD4 in the MON cell line. MON cell line, which was a gift from Dr. Delattre (Institute Curie, France), was established from a human MRT of soft tissue. ${ }^{31,32}$ Prior to the genome edition, TEAD4 copy number amplification in MON cell line was confirmed by Q-PCR (Fig. 3A). The cells were maintained in HyClone RPMI 1640 (Thermo Fisher Scientific) with $10 \%$ of $\mathrm{FBS}$ and penicillin/streptomycin at $37^{\circ} \mathrm{C}, 5 \% \mathrm{CO}_{2}$.

Lentiviral-CRISPR/Cas9 particles (Sigma-Aldrich, USA) were used for targeted genome editing. gRNA for HPRT was used as a positive control, and scrambled gRNA was used as a negative control. After transduction, cells were selected with puromycin for 14 days. The transduction efficiencies were confirmed with GeneArt Genomic Cleavage Detection kit (Life technologies) in order to detect the locusspecific double-strand break formation and to verify the efficiency of the genome edition.

\subsection{0 | Cell proliferation assay}

Cellular proliferation was assessed by TACS MTT Cell Proliferation Assays (Trevigen, USA) according to the manufacture's protocol. Absorbance was measured at $540 \mathrm{~nm}$ using a microplate reader after 24, 48, 72, and $96 \mathrm{hr}$. Each experiment was performed in triplicate. We also evaluated cell proliferative activity by IHC. Positive cells for $\mathrm{Ki}-67$ and $\mathrm{PHH} 3$ were counted in five fields with $40 \times$ magnification in both wild-type (WT) MON cells and TEAD4-mutated MON cells (TEAD4-mut).

\subsection{1 | Cell migration assay}

Cell migration was assessed using a 24-well Transwell chamber system (Corning, USA). ${ }^{33}$ After $24 \mathrm{hr}$ of incubation, the cells were fixed with formalin, stained by cresyl violet, and counted using an inverted microscope. Each experiment was performed in triplicate.

\section{3 | RESULTS}

\subsection{Tumor samples and primary cell cultures}

Primary AT/RT samples from 29 patients (the median age at diagnosis was 3 years with range from 2 months to 13 years, $M: F=13: 7$ )

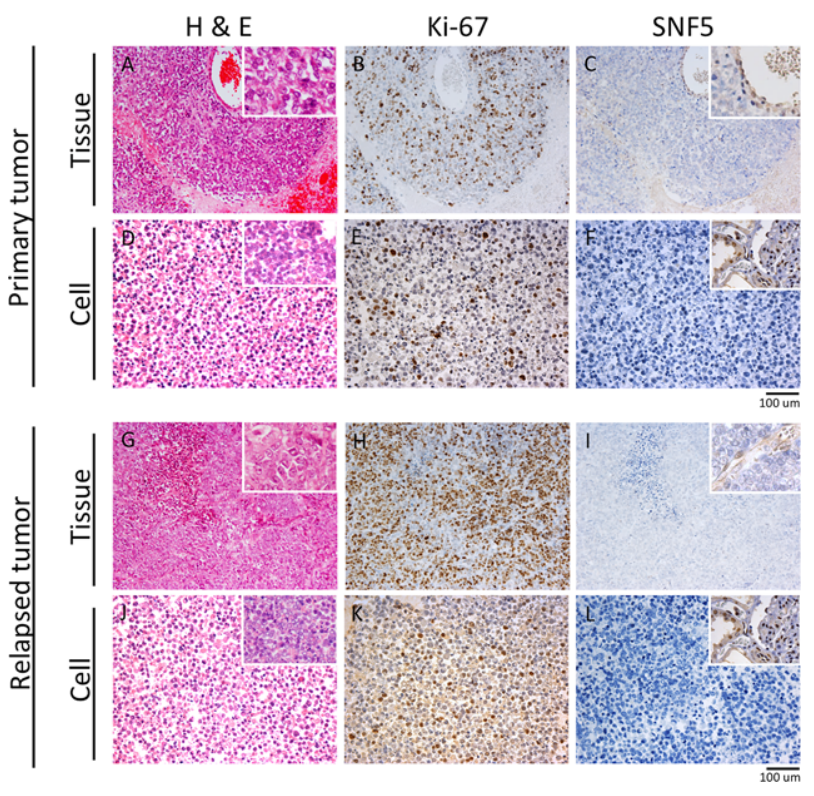

FIGURE 1 Establishment of primary cell cultures derived from primary and relapsed tumor tissue. (A and G) Hematoxylin and eosin (H\&E) staining revealed the presence of small undifferentiated cells and focal fields of rhabdoid cells in primary and relapsed tumor tissues. Characteristic rhabdoid cells were observed in insets. ( $B$ and $H$ ) High percentage for Ki-67-positive cells $(>50 \%)$ is observed in both primary and relapse tumors. (C and I) Nuclear immunostaining of SMARCB1 (BAF47/hSNF5/INI-1) was absent. Positive internal control was demonstrated in the insets. ( $D$ and $J$ ) $H \& E$ staining of cultured cells revealed the presence of small undifferentiated cells and focal fields of rhabdoid cells demonstrating that cultured cells have similar morphology of the corresponding primary tumor tissues. ( $E$ and $K$ ) High proliferative status of cultured cells as demonstrated by positive Ki-67 immunostaining ( $>50 \%$ ). ( $F$ and $L$ ) Nuclear immunostaining of SMARCB1 was absent in both primary and relapsed tissue derived cells. Normal kidney tissue was stained at the same time as a positive control for SMARCB1 (upper right) (all images: 40x; insets: 160x digital)

and additional four samples from corresponding relapsed tumor tissues were included in this study as described in Table 1. Fifteen MB samples were included in the study (the mean age at diagnosis was 5 years with range from 0 year to 10 years, $M: F=10: 5$ ).

Primary cell lines were established from tumors of patient number 1, who was a 6-month-old female having a tumor in the posterior fossa. Two months after near total removal, the tumor relapsed during the course of high-dose chemotherapy. Radiation therapy was performed after the second surgery, but the patient died of disease progression 6 months after her admission. From the first and second surgeries, primary cell lines were established. Hematoxylin and eosin stained slides revealed extensive areas of small undifferentiated cells and focal fields of rhabdoid cells (Figs. 1A, 1D, 1G, and 1J). In regards to the primary cell lines, the morphological features observed on cell block sections were consistent with the histology of the original tumor tissue sections. The diagnoses of AT/RT were further corroborated by loss of SMARCB1 nuclear expression in tumor cells with the presence of an appropriate internal positive control (Figs. 1C and 1I). Both sections of cell blocks from established cell lines also showed 
loss of SMARCB1 nuclear expression (Figs. 1F and 1L). High proliferative activity as detected by $\mathrm{IHC}$ for $\mathrm{Ki}-67$ was also demonstrated in all AT/RT samples and cell lines (Figs. 1B, 1E, 1H, and $1 K$ ).

\subsection{TEAD4 and YAP1 overexpression in primary cell lines}

CNVs were analyzed in the two primary cell lines, generated from primary and relapsed tumors, and in the correspondent relapsed tumor tissue. The chromosomal regions showing amplifications or deletions with more than twofold difference were selected. A total of 31 amplification sites and 3 deletion sites were detected. Three genes including TEAD4 were amplified within all the samples (Supplementary Table S1).

Then, the mRNA levels of TEAD4 and its co-activator YAP1 were investigated by In situ hybridization. Both primary and relapsed tumor tissues had significantly higher expression of TEAD4 and YAP1 when compared to normal brain tissue, but no differences between primary and relapsed tumors were observed $(P=0.0055$ and $P<0.0001$, respectively, one-way ANOVA) (Fig. 2A).

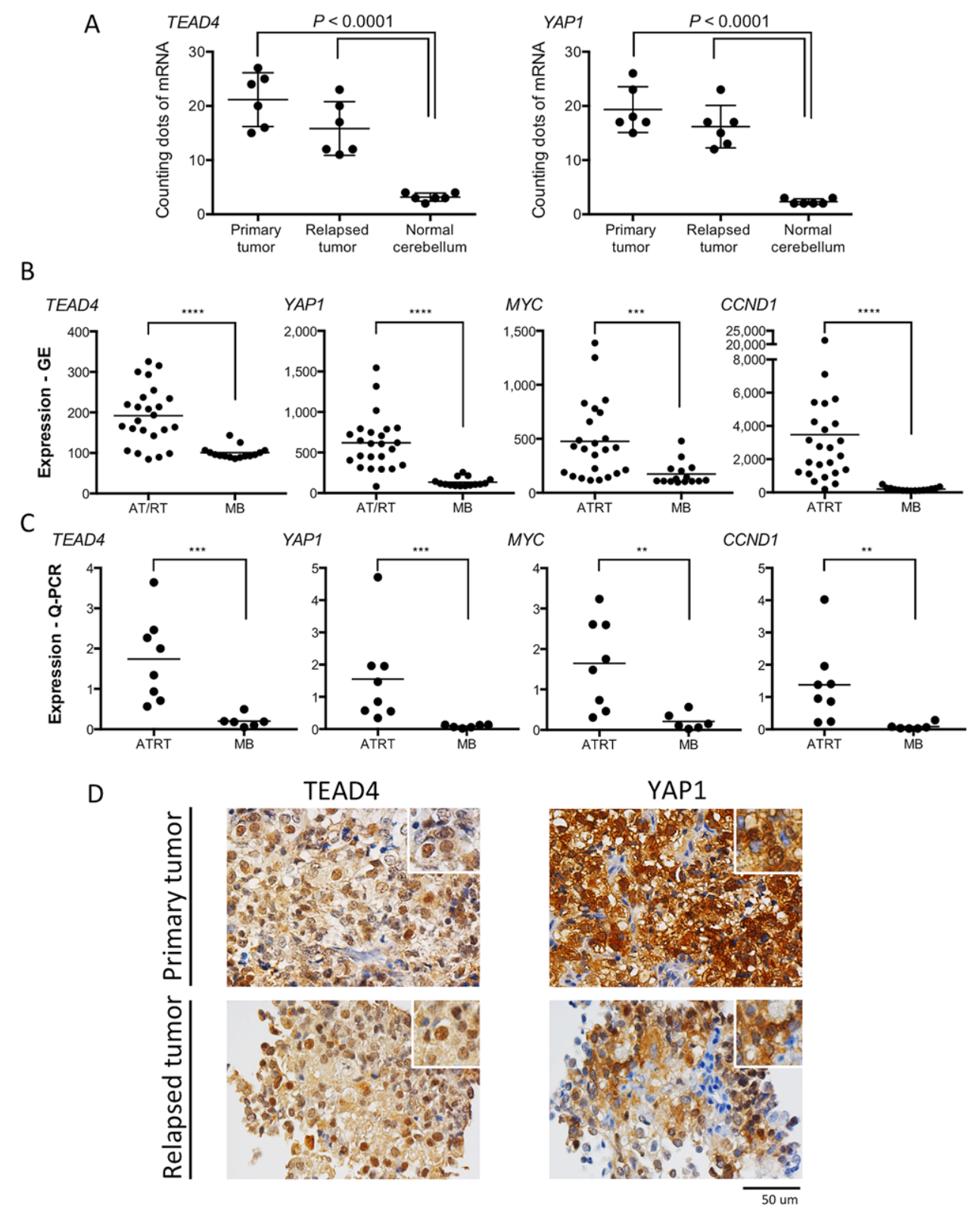

FIGURE 2 Overexpression of TEAD4 and YAP1 in AT/RT. (A) In situ hybridization for TEAD4 and YAP1 on samples from patient number 1. The results were analyzed by counting existing dots of mRNA and comparing tumor cells to surrounding normal cells. TEAD4 and YAP1 showed significantly higher expression in tumors, for both primary and relapse when compared with normal cerebellum $(P=0.0055$ and $P<0.0001$, respectively, one-way ANOVA). (B) Gene expression analysis showed significantly higher expression of TEAD4, YAP1, MYC, and CCND1 in AT/RT when compared to $\mathrm{MB}$ (fold changes $=1.95,5.56,3.56$, and 15.7 , respectively; ${ }^{* * *} P<0.0001$ and ${ }^{* * *} P=0.0001$, Mann-Whitney test). (C) Microarray gene expression data were validated by quantitative real-time PCR. All of the above genes were significantly overexpressed in AT/RT (fold changes $=8.62,17.2$, 7.91, and 16.2, respectively; ${ }^{* *} P<0.001$ and ${ }^{* *} P<0.01$, Mann-Whitney test). (D) Immunohistochemistry for TEAD4 and YAP1 from samples of patient number 1 (40x; inset: 160x digital). TEAD4 and YAP1 were highly expressed in AT/RT both primary and relapsed tumor tissues. TEAD4 was localized in nuclei and YAP1 was localized in both cytoplasm and nuclei 
A

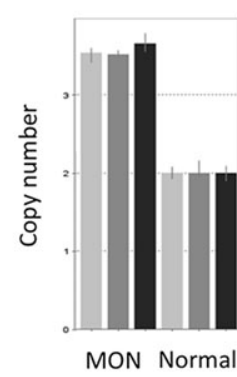

B

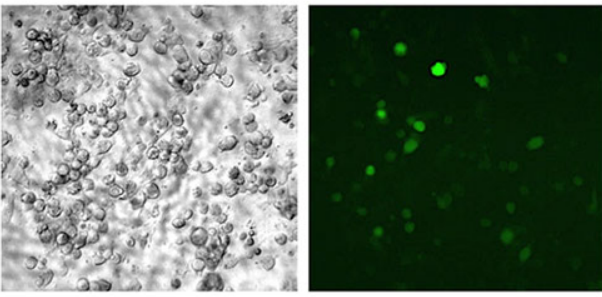

D

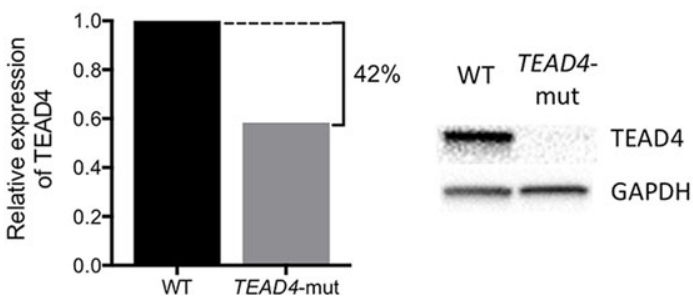

FIGURE 3 TEAD4-mutated MRT cell line. (A) Copy number amplification of TEAD4 is observed in MON cell line. Normal blood cells were used as control. (B) Posttransduced images (10x, right; bright field, left; fluorescent microscope). GFP-positive cells indicated the efficiency of transduction: 22.3\%. (C) GCD assay showed two cleaved bands below the parental band in TEAD4-mutated cells, confirming genome editing. Following two sets of primers were designed. Primer 1: $3^{\prime}$-TGTGATCCAGAGAGGGAACC and 5'-CATTGAACCCAGGAGGAGA; primer 2: 3'-TGTGATCCAGAGAGGGAACC and 5'-TCACTTGAACCCAGGAGGAG. (D) Q-PCR shows $42 \%$ reduction of TEAD4 expression in mutated cells and western blotting demonstrates that TEAD4 expression was suppressed in mutated cells despite the equal level of endogenous control, GAPDH

\section{3 | Verification of TEAD4 and YAP1 overexpression in an independent set of samples}

To verify the TEAD4 and YAP1 overexpression, GE profiling was evaluated in an independent set of $24 \mathrm{AT} / \mathrm{RT}$ and $15 \mathrm{MB}$ samples. The expression of both TEAD4 and YAP1 was significantly higher in AT/RT (fold changes $=1.95$ and 5.56, respectively; $P<0.0001$, MannWhitney test) (Fig. 2B). The correlation between expression levels and molecular subgroups, as defined by Torchia et al. ${ }^{16}$ and Johann et al., ${ }^{17}$ was investigated. TEAD4 expression in Torchia's group 1 was significantly lower than in group $2(P<0.0001)$. Johann's ATRT-SHH showed a tendency to express TEAD4 in lower levels when compared to ATRTTYR and ATRT-MYC. YAP1 was also less expressed in Torchia's group 1 and Johann's ATRT-SHH. The expression levels of both TEAD4 and YAP1 did not show correlation with age, gender, or tumor location.

The expression levels of all components of the Hippo signaling pathway according to KEGG were investigated. MST and LATS, upstream kinases of the pathway, did not show differences in expression between AT/RT and MB. The expression of MER, KIBRA, and FRMD, which are considered to be regulators of these kinases even though their function in the Hippo pathway has not been revealed yet, also did not show differential expression. The downstream targets of the Hippo pathway, MYC and CCND1, were significantly overexpressed in AT/RT (fold changes $=3.56$ and $15.7 ; P=0.0001$ and $P<0.0001$, respectively, Mann-Whitney test) (Fig. 2B). GE profiling was validated by Q-PCR in $8 \mathrm{AT} / \mathrm{RT}$ and $6 \mathrm{MB}$ samples (fold changes = 1.95, 5.56, 3.56, and 15.7; $P=0.0007,0.0007,0.0027$, and 0.0047 , respectively, Mann-Whitney test) (Fig. 2C).
The protein expression of TEAD4 and YAP1 was investigated by IHC in 16 FFPE samples of 12 patients (Table 1). All sections including primary and relapsed tumor tissues, showed high expression of both TEAD4 and YAP1. TEAD4 was expressed almost exclusively in the nuclei, while YAP1 expression was observed both in the cytoplasm and nuclei of tumor cells. Neither the intensity of expression nor the localization of both proteins differed between primary and relapsed tumors (Fig. 2D)

\subsection{TEAD4-mutated MRT cell line by CRISPR/Cas9}

We used Lentiviral-CRISPR/Cas9 system to mutate TEAD4 in the MON cell line. Transfection efficacy was estimated by the percentage of GFPpositive cells (Fig. 3B) and genome edition was confirmed by GCD (genome cleavage detection) assay (Fig. 3C). The result from Q-PCR showed $42 \%$ reduction of mRNA level of TEAD4 in mutated cells, and protein level was evaluated by western blotting (Fig. 3D).

\subsection{Decreased cell proliferation and migration in TEAD4-mutated MRT cells}

Cellular proliferation was assessed by MTT assay and IHC for Ki67 and $\mathrm{PHH} 3$ antibodies. MTT assay showed statistically significant decrease in cell proliferation in TEAD4-mut when compared with WT at all time points $(P=0.0020,0.0021,0.0025$, and 0.0193 , respectively, unpaired $t$-test) (Fig. 4A). IHC for Ki-67 showed high proliferation in both cell lines. However, there were significantly less Ki67positive cells within TEAD4-mut than within WT $(P=0.0021$, unpaired 
A

\section{Cell proliferation curve}

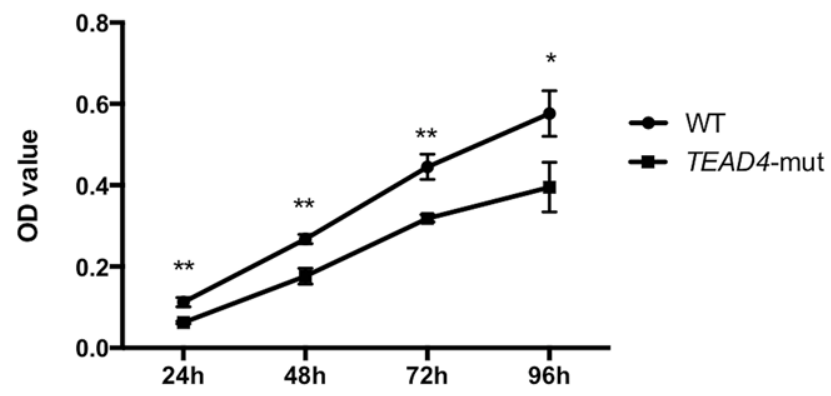

B
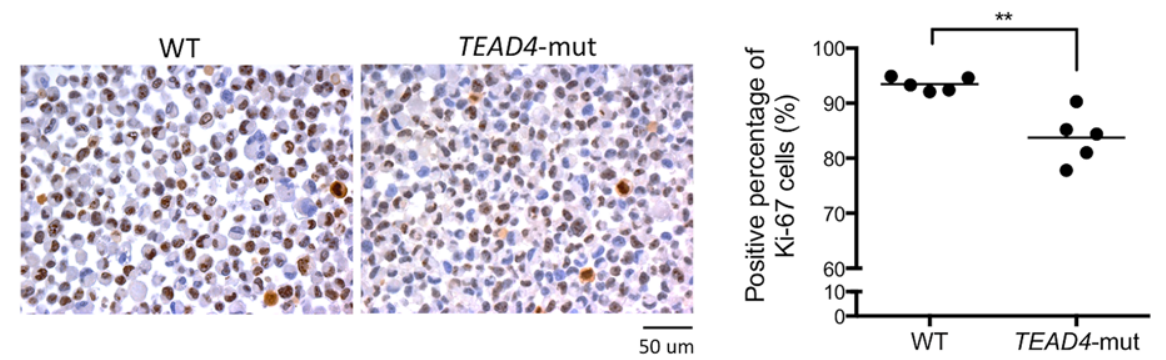

C
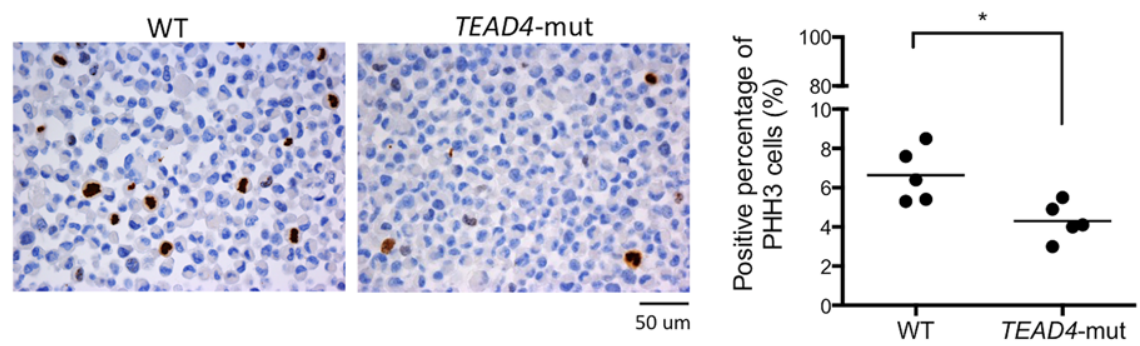

D
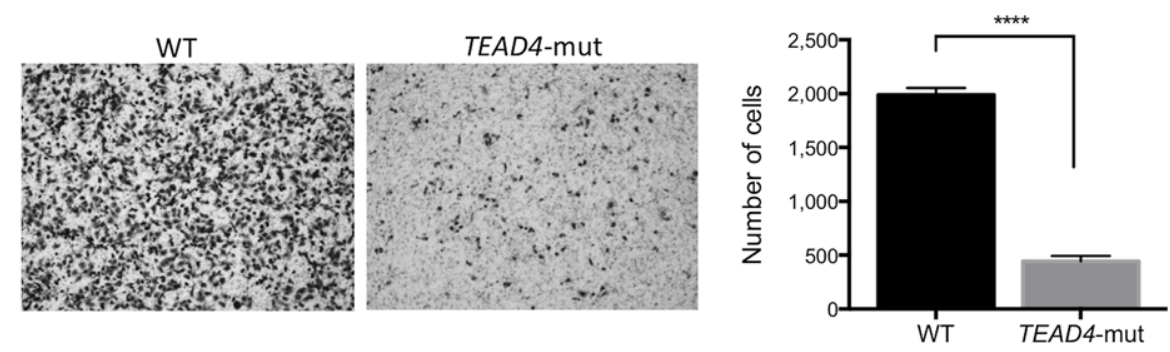

FIGURE 4 Cell proliferation was decreased in TEAD4-mutated MRT cell line. (A) MTT proliferation assay: significantly lower cell proliferation was observed in TEAD4-mutated cells when compared to wild-type (unpaired $t$-test; ${ }^{*} P<0.05,{ }^{* *} P<0.005$ ). (B) Representative images and analysis of Ki-67 (40x): positive percentage was significantly higher in wild-type (unpaired t-test; $\left.{ }^{* *} P<0.005\right)$. (C) Representative images and analysis of $\mathrm{PHH} 3$ antibody (40X): mitotic activity was detected by $\mathrm{PHH} 3$ staining. TEAD4-mutated cells showed significantly lower mitotic activity (unpaired $t$-test; $\left.{ }^{*} P<0.05\right)$. (D) Transwell migration assay: significantly lower migration ability was observed in TEAD4-mutated cells (unpaired $t$-test, $\left.{ }^{* * * *} P<0.0001\right)$

t-test) (Fig. 4B). Mitotic activity, measured by $\mathrm{PHH} 3$ antibody, was also significantly lower in the TEAD4-mut $(P=0.0147$, unpaired $t$ test) (Fig. 4C). Notably, cellular migration was significantly inhibited in TEAD4-mut when compared with WT $(P<0.0001$, unpaired $t$-test) (Fig. 4D)

\section{4 | DISCUSSION}

In this study, we report our finding of copy number amplification of TEAD4 and explore the overexpression of TEAD4 and YAP1 in AT/RT.
TEAD4 and YAP1 are the key components of Hippo signaling pathway, which has been recognized as a tumor suppressor pathway in recent years. ${ }^{23,34-36}$ We revealed the copy number amplification of TEAD4 in primary cell lines and correspondent relapsed tissue from a patient. Then, we confirmed the overexpression of TEAD4 and its co-activator YAP1 at mRNA level and at protein level in the same patient's samples. Finally, we validated our findings in an independent cohort of samples. To the best of our best knowledge, this is the first time TEAD4 overexpression is reported in AT/RT.

In normal cells, polarity and adhesion complexes regulate the Hippo pathway and the pathway controls organ size and 
regeneration through the inhibition of cell proliferation and promotion of apoptosis, ${ }^{37-39}$ while in several human cancers, this pathway is dysregulated and this dysregulation is supposed to contribute to cancer development. Although many publications report upregulation of YAP1, only a few reports describe upregulation of TEAD4 in cancer. ${ }^{24,26,29}$ Liu et al. reported that in colorectal cancer, increased TEAD4 expression is a result of copy number amplification. ${ }^{29} \mathrm{We}$ also observed copy number amplification and overexpression at mRNA and protein levels of TEAD4 in AT/RT.

Since YAP1 cannot bind to DNA by itself, the YAP1 protein in the nuclei is required to be co-localized with TEAD4 for the oncogenic activation of YAP1. ${ }^{23}$ The co-localization of TEAD4 and YAP1 in nuclei has been correlated with poor prognosis in human malignancies such as ovarian cancer and gastric cancer. ${ }^{24,26}$ In this study we compared $\mathrm{AT} / \mathrm{RT}$ to $\mathrm{MB}$, which is the most common pediatric embryonal tumor in the CNS and has a better outcome than AT/RT, with over $90 \%$ of cure rates for WNT group and $40-60 \%$ for group $3 .{ }^{40,41}$ Due to the insufficient clinical information, we could not analyze the correlation between the expression levels and the clinical outcome in our AT/RT cohort.

Lim et al. reported that the knockdown of TEAD4 resulted in the reduced growth of gastric cancer cells in vitro and in vivo. ${ }^{24}$ In this study we knocked down TEAD4 in the MON cell line, which is a wellcharacterized MRT cell line, and we observed both decrease in proliferation and inhibition of migration of TEAD4-mut rhabdoid cells. Although our results are in accordance with the literature, we appreciate the fact that the use of MON may be somehow controversial. While some authors suggested that the differences between MRTs arising in different locations are minimal, ${ }^{1}$ other investigator demonstrated low overlap in GE of AT/RT and RTK. ${ }^{42}$ Knocking down TEAD4 in an AT/RT cell line may clarify this question. Based on these findings, we suggest that TEAD4, together with its co-activator YAP1, functions as oncogenes and may contribute to the biology of AT/RT.

Overexpression of MYC and CCND1 has been extensively reported in AT/RT. 43,44 They are already well-known protooncogenes and also downstream targets of the Hippo pathway. On the other hand, both MYC and CCND1 are part of the Wnt pathway that is known to be dysregulated in a subset of AT/RT. ${ }^{45}$ Recently, Johann et al. proposed the existence of three AT/RT epigenetic subgroups: ATRT-TYR, ATRT-SHH, and ATRT-MYC. Each of these groups has different clinical characteristics and subgroup-specific networks, granting the possibility of therapeutic intervention. ${ }^{17}$ MYC overexpression is the marker of ATRT-MYC and CCND1 was proposed to be the specific enhancer for ATRT-TYR subgroup. Neither TEAD4 nor YAP1 is included in the genetic signatures or networks proposed by the authors. We used their classification system to categorize our 24 AT/RT samples, and observed that ATRT-SHH has a tendency to express TEAD4 and YAP1 at lower levels. Another molecular classification was proposed by Torchia et al. taking into consideration anatomical location, clinical features, and the level of ASCL1, a gene involved in the Notch signaling pathway. ${ }^{16} \mathrm{We}$ observed that group $1 \mathrm{AT} / \mathrm{RT}$, that is, ASCL1-positive, had significantly lower expression of TEAD4 when compared to group 2. No difference in YAP1 was observed. ${ }^{46}$ CCND1 was overexpressed in all our samples. We cannot affirm, based on our results, that overexpression of MYC and CCND1 is a direct response of TEAD4 activation, as no other component of the pathway was found to be differentially expressed in our samples. Furthermore, it is supposed that other cancer-related pathways, such as TGF- $\beta$ signaling pathway and Wnt signaling pathway, also regulate the downstream targets of the Hippo pathway. Further studies are needed to investigate the effects of TEAD4 activation on the Hippo pathway in AT/RT.

In conclusion, we report the overexpression of TEAD4 in AT/RT. High TEAD4 expression was observed in all our cases, including primary and relapsed tumors, at both mRNA and protein levels. Moreover, our results showed that TEAD4 knockdown significantly impaired proliferative activity in vitro. It is feasible to therapeutically target AT/RT by inhibiting the interaction between YAP1 and TEAD4, as has been done in other human cancers. ${ }^{35}$ Although TEAD4 may be an essential component of AT/RT biology, further studies are needed to explore the functional significance of these findings and whether the Hippo pathway is an essential component of AT/RT biology.

\section{ACKNOWLEDGMENTS}

This work was supported by Graduate School Research Program from Juntendo University, Juntendo University Research Institute for Disease of Old Ages, and the Rally Foundation for Childhood Cancer Research in memory of Hailey Trainer. We thank Miyuki Kunichika, Naira Margarian DVM, PhD, Lin Li MD, MSc, and Dorina Veliceasa, $\mathrm{PhD}$, for technical assistance, and Jessica Jacubowski, BSc, for editorial support. We also thank all the patients, their families, and the people who work for our patients.

\section{CONFLICT OF INTEREST}

The authors declare that there is no conflict of interest.

\section{REFERENCES}

1. Grupenmacher AT, Halpern AL, Bonaldo Mde F, et al. Study of the gene expression and microRNA expression profiles of malignant rhabdoid tumors originated in the brain (AT/RT) and in the kidney (RTK). Childs Nerv Syst. 2013;29:1977-1983.

2. Woehrer A, Slavc I, Waldhoer T, et al. Incidence of atypical teratoid/rhabdoid tumors in children: a population-based study by the Austrian Brain Tumor Registry, 1996-2006. Cancer. 2010;116:57255732.

3. Ginn KF, Gajjar A. Atypical teratoid rhabdoid tumor: current therapy and future directions. Front Oncol. 2012;2:114. doi: 10.3389/fonc.2012.00114

4. Athale UH, Duckworth J, Odame I, Barr R. Childhood atypical teratoid rhabdoid tumor of the central nervous system: a metaanalysis of observational studies. J Pediatr Hematol Oncol. 2009;31: 651-663.

5. von Hoff K, Hinkes B, Dannenmann-Stern E, et al. Frequency, riskfactors and survival of children with atypical teratoid rhabdoid tumors (AT/RT) of the CNS diagnosed between 1988 and 2004, and registered to the German HIT database. Pediatr Blood Cancer. 2011;57: 978-985.

6. Benesch M, Bartelheim K, Fleischhack G, et al. High-dose chemotherapy (HDCT) with auto-SCT in children with atypical 
teratoid/rhabdoid tumors (AT/RT): a report from the European Rhabdoid Registry (EU-RHAB). Bone Marrow Transplant. 2014;49: 370-375.

7. Lafay-Cousin L, Hawkins C, Carret AS, et al. Central nervous system atypical teratoid rhabdoid tumours: the Canadian Paediatric Brain Tumour Consortium experience. Eur J Cancer. 2012;48: 353-359.

8. Slavc I, Chocholous M, Leiss U, et al. Atypical teratoid rhabdoid tumor: improved long-term survival with an intensive multimodal therapy and delayed radiotherapy. The Medical University of Vienna Experience 1992-2012. Cancer Med. 2014;3:91-100.

9. Zaky W, Dhall G, Ji L, et al. Intensive induction chemotherapy followed by myeloablative chemotherapy with autologous hematopoietic progenitor cell rescue for young children newly-diagnosed with central nervous system atypical teratoid/rhabdoid tumors: the Head Start III experience. Pediatr Blood Cancer. 2014;61: 95-101.

10. DiPatri AJ, Jr., Sredni ST, Grahovac G, Tomita T. Atypical teratoid rhabdoid tumors of the posterior fossa in children. Childs Nerv Syst. 2015;31:1717-1728.

11. Louis DN, Ohgaki H, Wiestler OD, Cavenee WK. WHO Classification of Tumours of the Central Nervous System. 4th ed.; World Health Organization, 2007.

12. Louis DN, Perry A, Reifenberger G, et al. The 2016 World Health Organization Classification of Tumors of the Central Nervous System: a summary. Acta Neuropathol. 2016;131:803-820.

13. Biegel JA, Zhou JY, Rorke LB, et al. Germ-line and acquired mutations of INI1 in atypical teratoid and rhabdoid tumors. Cancer Res. 1999;59:74-79.

14. Judkins AR, Mauger J, Ht A, Rorke LB, Biegel JA. Immunohistochemical analysis of hSNF5/INI1 in pediatric CNS neoplasms. Am J Surg Pathol. 2004;28:644-650.

15. Birks DK, Donson AM, Patel PR, et al. High expression of BMP pathway genes distinguishes a subset of atypical teratoid/rhabdoid tumors associated with shorter survival. Neuro Oncol. 2011;13: 1296-1307.

16. Torchia J, Picard D, Lafay-Cousin L, et al. Molecular subgroups of atypical teratoid rhabdoid tumours in children: an integrated genomic and clinicopathological analysis. Lancet Oncol. 2015;16: 569-582.

17. Johann PD, Erkek S, Zapatka M, et al. Atypical teratoid/rhabdoid tumors are comprised of three epigenetic subgroups with distinct enhancer landscapes. Cancer Cell. 2016;29:379-393.

18. Lee S, Cimica V, Ramachandra N, Zagzag D, Kalpana GV. Aurora A is a repressed effector target of the chromatin remodeling protein INI1/hSNF5 required for rhabdoid tumor cell survival. Cancer Res. 2011;71:3225-3235.

19. Smith ME, Cimica V, Chinni S, et al. Therapeutically targeting cyclin D1 in primary tumors arising from loss of Ini1. Proc Natl Acad Sci USA. 2011;108:319-324.

20. Alimova I, Birks DK, Harris PS, et al. Inhibition of EZH2 suppresses selfrenewal and induces radiation sensitivity in atypical rhabdoid teratoid tumor cells. Neuro Oncol. 2013;15:149-160.

21. Sredni ST, Patel K, D’Almeida Costa F, de Fatima Bonaldo M, Tomita T. Activation of ErbB2- ErbB3 signaling pathway supports potential therapeutic activity of ErbB inhibitors in AT/RT. J Neurooncol. 2014;118:201-203.

22. Fruhwald MC, Biegel JA, Bourdeaut F, Roberts CW, Chi SN. Atypical teratoid/rhabdoid tumors-current concepts, advances in biology, and potential future therapies. Neuro Oncol. 2016;18:764-778.
23. Nishio M, Otsubo K, Maehama T, Mimori K, Suzuki A. Capturing the mammalian Hippo: elucidating its role in cancer. Cancer Sci. 2013;104:1271-1277.

24. Lim B, Park JL, Kim HJ, et al. Integrative genomics analysis reveals the multilevel dysregulation and oncogenic characteristics of TEAD4 in gastric cancer. Carcinogenesis. 2014;35:1020-1027.

25. Johnson R, Halder G. The two faces of Hippo: targeting the Hippo pathway for regenerative medicine and cancer treatment. Nat Rev. 2014;13:63-79.

26. Xia Y, Chang T, Wang Y, et al. YAP promotes ovarian cancer cell tumorigenesis and is indicative of a poor prognosis for ovarian cancer patients. PLoS One. 2014;9:e91770.

27. Yuan H, Liu H, Liu Z, et al. Genetic variants in Hippo pathway genes YAP1, TEAD1 and TEAD4 are associated with melanoma-specific survival. Int J Cancer. 2015;137:638-645.

28. Wang C, Nie Z, Zhou Z, et al. The interplay between TEAD4 and KLF5 promotes breast cancer partially through inhibiting the transcription of p27Kip1. Oncotarget. 2015;6:17685-17697.

29. Liu Y, Wang G, Yang Y, et al. Increased TEAD4 expression and nuclear localization in colorectal cancer promote epithelial-mesenchymal transition and metastasis in a YAP-independent manner. Oncogene. 2016;35:2789-2800.

30. Xu J, Erdreich-Epstein A, Gonzalez-Gomez I, et al. Novel cell lines established from pediatric brain tumors. J Neurooncol. 2012;107:269280.

31. Zhang ZK, Davies KP, Allen J, et al. Cell cycle arrest and repression of cyclin D1 transcription by INI1/hSNF5. Mol Cell Biol. 2002;22:59755988.

32. Versteege I, Medjkane S, Rouillard D, Delattre O. A key role of the hSNF5/INI1 tumour suppressor in the control of the G1-S transition of the cell cycle. Oncogene. 2002;21:6403-6412.

33. Zheng B, Liang L, Huang S, et al. MicroRNA-409 suppresses tumour cell invasion and metastasis by directly targeting radixin in gastric cancers. Oncogene. 2012;31:4509-4516.

34. Lamar JM, Stern P, Liu H, et al. The Hippo pathway target, YAP, promotes metastasis through its TEAD-interaction domain. Proc Natl Acad Sci USA. 2012;109:E2441-E2450.

35. Johnson R, Halder G. The two faces of Hippo: targeting the Hippo pathway for regenerative medicine and cancer treatment. Nat Rev Drug Discov. 2014;13:63-79.

36. Chan SW, Lim CJ, Chen L, et al. The Hippo pathway in biological control and cancer development. J Cell Physiol. 2011;226:928-939.

37. Ota M, Sasaki H. Mammalian TEAD proteins regulate cell proliferation and contact inhibition as transcriptional mediators of Hippo signaling. Development. 2008;135:4059-4069.

38. Zhao B, Tumaneng K, Guan KL. The Hippo pathway in organ size control, tissue regeneration and stem cell self-renewal. Nat Cell Biol. 2011;13:877-883.

39. Zhou Y, Huang T, Cheng AS, et al. The TEAD family and its oncogenic role in promoting tumorigenesis. Int J Mol Sci. 2016;17:138.

40. Massimino M, Biassoni V, Gandola L, et al. Childhood medulloblastoma. Crit Rev Oncol Hematol. 2016;105:35-51.

41. Coluccia D, Figuereido C, Isik S, Smith C, Rutka JT. Medulloblastoma: tumor biology and relevance to treatment and prognosis paradigm. Curr Neurol Neurosci Rep. 2016;16:43.

42. Birks DK, Donson AM, Patel PR, et al. Pediatric rhabdoid tumors of kidney and brain show many differences in gene expression but share dysregulation of cell cycle and epigenetic effector genes. Pediatr Blood Cancer. 2013;60:1095-1102. 
43. Fujisawa H, Misaki K, Takabatake $\mathrm{Y}$, Hasegawa M, Yamashita J. Cyclin D1 is overexpressed in atypical teratoid/rhabdoid tumor with hSNF5/INI1 gene inactivation. J Neurooncol. 2005;73: 117-124.

44. Hanahan D, Weinberg RA. The hallmarks of cancer. Cell. 2000;100:5770.

45. Chakravadhanula M, Hampton CN, Chodavadia P, et al. Wnt pathway in atypical teratoid rhabdoid tumors. Neuro Oncol. 2015;17:526-535.

46. Fernandez LA, Northcott PA, Dalton J, et al. YAP1 is amplified and up-regulated in hedgehog-associated medulloblastomas and mediates sonic hedgehog-driven neural precursor proliferation. Genes Dev. 2009;23:2729-2741.

\section{SUPPORTING INFORMATION}

Additional Supporting Information may be found online in the supporting information tab for this article.

How to cite this article: Suzuki M, Kondo A, Ogino I, Arai H, Tomita T, Sredni ST. Overexpression of TEAD4 in atypical teratoid/rhabdoid tumor: New insight to the pathophysiology of an aggressive brain tumor. Pediatr Blood Cancer. 2017;64:e26398. DOI: $10.1002 / p b c .26398$ 